\title{
Double-blind holography of attosecond pulses
}

\author{
O. Pedatzur' ${ }^{1 \star}$, A. Trabattoni ${ }^{2,3}$, B. Leshem ${ }^{1}$, H. Shalmoni', M. C. Castrovilli ${ }^{4,5}$, M. Galli ${ }^{2,4}$, M. Lucchini ${ }^{2,4}$, \\ E. Månsson $\oplus^{4}{ }^{4}$, F. Frassetto ${ }^{6}$, L. Poletto ${ }^{6}$, B. Nadler ${ }^{7}$, O. Raz ${ }^{1}$, M. Nisoli ${ }^{2,4}$, F. Calegari ${ }^{3,4,8}$, D. Oron ${ }^{1}$ and \\ N. Dudovich ${ }^{1 *}{ }^{\text {* }}$
}

\begin{abstract}
A key challenge in attosecond science is the temporal characterization of attosecond pulses that are essential for understanding the evolution of electronic wavefunctions in atoms, molecules and solids ${ }^{1-7}$. Current characterization methods, based on nonlinear light-matter interactions, are limited in terms of stability and waveform complexity. Here, we experimentally demonstrate a conceptually new linear and alloptical pulse characterization method, inspired by doubleblind holography. Holography is realized by measuring the extreme ultraviolet (XUV) spectra of two unknown attosecond signals and their interference. Assuming a finite pulse duration constraint, we reconstruct the missing spectral phases and characterize the unknown signals in both isolated pulse and double pulse scenarios. This method can be implemented in a wide range of experimental realizations, enabling the study of complex electron dynamics via a single-shot and linear measurement.
\end{abstract}

Isolated attosecond pulses are unique tools for studying the natural timescale of electronic processes within matter. A fundamental building block in attosecond time-resolved spectroscopy is the ability to precisely characterize their temporal shape. These pulses encode valuable structural and dynamical information, combining attosecond timing accuracy with ångström spatial precision. This information is of key importance for understanding the evolution of electronic wavefunctions in atoms ${ }^{1}$, molecules ${ }^{2-6}$ and solids ${ }^{7}$. Studying such phenomena requires the development of a complete and robust characterization method for attosecond pulses, posing one of the primary challenges in this field.

Clearly, the short duration of attosecond pulses, well below the temporal resolution of detector electronics, does not allow for direct characterization in the time domain. Therefore, their measurement is performed in the spectral domain, but this leads to the loss of spectral phase information. One approach to recover the lost spectral information is to measure the interference of different frequency components. However, as such an interference cannot be obtained via linear measurements, it requires the nonlinear light-matter interaction. Commonly used methods, such as FROG-CRAB (frequency resolved optical gating for complete reconstruction of attosecond bursts) ${ }^{8,9}$ and RABBITT (reconstruction of attosecond beating by interference of two-photon transitions) $)^{1,10}$ involve a nonlinear interaction of the attosecond pulse with the near-infrared (NIR) fundamental field. Interferometric autocorrelation can be achieved via the use of an XUV-XUV nonlinear interaction ${ }^{11,12}$. Finally, the measurement can rely on the intrinsic nonlinearity of the production process itself ${ }^{13,14}$. Yet, all these time-resolved nonlinear measurements require repetitive measurements at various delays between multiple fields, and are sensitive to noise. Due to the limitations of the available techniques, some state-of-the-art attosecond sources, such as X-ray free-electron lasers, are difficult to characterize adequately.

In this work we experimentally demonstrate a conceptually different approach to directly measure and characterize XUV attosecond pulses. In contrast with commonly used femtosecond and attosecond characterization methods, our method is based on a linear measurement of the XUV spectrum, obviating the need to mix different XUV frequencies. This approach, termed doubleblind holography $(\mathrm{DBH})$, relies on two key components. The first is perhaps the most fundamental property of attosecond pulses-a finite temporal duration, also known as a 'compact support' in the time domain. The second component is a spectral measurement of XUV signals from two independent coherent sources as well as their interference, defining a double-blind temporal hologram. Figure 1a depicts how such a 'temporal hologram' can be obtained, where each arm of the interferometer represents an independent attosecond source, while the spectral measurement acts as a beam combiner that resolves their interference. Temporal holograms can be realized in a range of experimental systems - from polarization measurements ${ }^{15}$ to two sources ${ }^{16}$ and even through multi-orbital contributions $^{3}$. In our work we implement this scheme using attosecond pulses generated in a mixture of two gases. We further demonstrate the ability to perform a single-shot realization of $\mathrm{DBH}$ via the interference between two time-delayed pulses.

Linear phase retrieval schemes have been extensively used in the spatial domain for the retrieval of objects from their diffraction patterns, as in X-ray lensless imaging or electron diffraction, where the retrieved object is two-dimensional (2D). Indeed, for $2 \mathrm{D}$ objects with finite support, a densely sampled diffraction pattern is sufficient for the reconstruction of phase information ${ }^{17-21}$. In contrast, the problem of reconstructing an ultrashort temporal $1 \mathrm{D}$ signal from its measured spectrum is ill posed, having multiple valid solutions ${ }^{22,23}$. Holography, another common phase measurement scheme, relies on a known reference signal. In holography, interference with a known signal maps the missing phase into intensity modulation of the measured signal. Yet, the generation of a well-characterized reference for attosecond temporal holography is extremely difficult. $\mathrm{DBH}$ is a scheme that combines a compact support constraint together with holography using an unknown reference, so as to overcome both their individual limitations. This

\footnotetext{
'Department of Physics of Complex Systems, Weizmann Institute of Science, Rehovot, Israel. ${ }^{2}$ Department of Physics, Politecnico di Milano, Milano, Italy. ${ }^{3}$ Center for Free-Electron Laser Science, DESY, Hamburg, Germany. ${ }^{4}$ Institute for Photonics and Nanotechnologies, CNR-IFN, Milano, Italy. ${ }^{5}$ Inst. for the Structure of Matter CNR-ISM, Monterotondo, Italy. ${ }^{6}$ Institute for Photonics and Nanotechnologies CNR-IFN, Padova, Italy. ${ }^{7}$ Department of Computer Science and Applied Mathematics, Weizmann Institute of Science, Rehovot, Israel. ${ }^{8}$ Department of Physics, Hamburg Universität, Hamburg, Germany. *e-mail: orenpedatzur@gmail.com; nirit.dudovich@weizmann.ac.il
} 


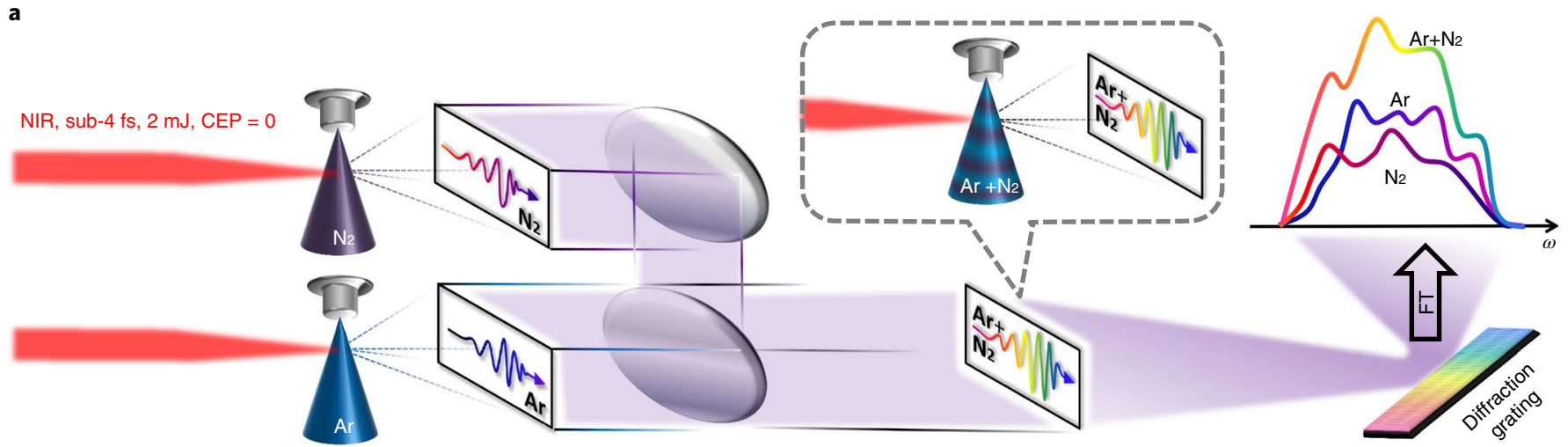

b

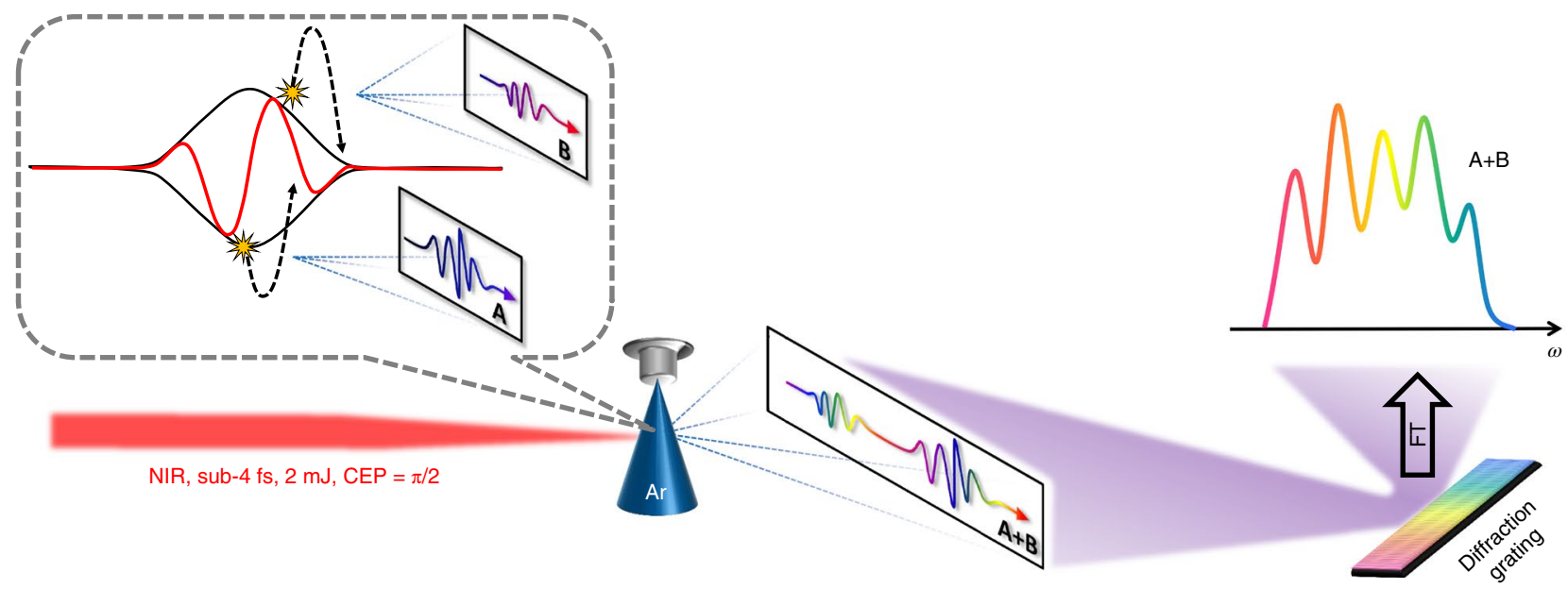

Fig. 1 | Schematic description of temporal DBH. a, Gas mixture DBH: the two arms of the hologram represent the XUV spectrum, produced from Ar and $\mathrm{N}_{2,}$ respectively. The spectral interference between the two arms is achieved by producing HHG in an $\mathrm{Ar}$ and $\mathrm{N}_{2}$ gas mixture. $\mathbf{b}$, Single-shot DBH: in a carrierenvelope phase (CEP) $\sim \pi / 2$ configuration, two consecutive half-cycles of the driving field produce two different attosecond pulses delayed by half a period of the NIR. Interference between these two pulses gives rise to spectral fringes. FT, Fourier transform.

concept has been applied in the spatial domain to retrieve the diffraction phase in coherent diffractive (lensless) imaging scenarios in the visible ${ }^{24}$ and $\mathrm{X}$-ray regimes ${ }^{25}$. Here, we apply this scheme in the temporal domain and demonstrate its ability to perform a linear reconstruction of isolated attosecond pulses.

In the following, we briefly describe the essence of the method. For a detailed theoretical study of this method, its advantages and limitations, see refs. ${ }^{26,27}$. Consider two discretized XUV spectra, each of length $N$. Their spectral phases define a set of $2 N$ unknowns, $X_{n}^{A}=e^{i \phi_{n}^{A}}$ and $X_{n}^{B}=e^{i \phi_{n}^{B}}$ with $n=0,1, \ldots, N-1$. A compact support constraint in the time domain requires that the Fourier transform of the pulse spectrum vanishes outside of the support. This constraint introduces a set of $2(N-T)$ linearly independent equations (equations (3) and (4) in the Methods), where $T$ is the width of the compact support. Measuring the spectral interference of the two pulses allows for direct reconstruction of their spectral phase difference, $\phi_{n}^{B}-\phi_{n}^{A}$, forming an additional set of $N$ linear equations (equations (1) and (2) in the Methods). Overall, these three measurements provide us with an excess of linear equations, which removes the degeneracy associated with the 1D classical phase retrieval problem, allowing its direct reconstruction ${ }^{26}$. Importantly, this method does not require a priori knowledge of the correct compact support. Instead, we scan over potential compact support widths and apply the DBH algorithm for each assumed one. In each iteration we calculate an error score (Methods, equation (7)), which quantifies the residual energy outside of the assumed compact support. The estimated compact support is the global minimum of this error score. Realistically, the spectral measurements are accompanied by additive noise. Furthermore, the temporal signals may have decaying tails that leak out of the compact support. Investigation of the quality of reconstruction in the presence of noise and for exponentially decaying signals can be found in ref. ${ }^{27}$.

We experimentally realized DBH of attosecond pulses using the following system. A sub- $4 \mathrm{fs}, 1 \mathrm{~mJ}, 800 \mathrm{~nm}$, carrier-envelope phase (CEP) stabilized pulse was focused into a gas cell. The pressure in the cell was regulated by a leak valve under different backing pressures: 3 bar $\mathrm{N}_{2}$ and 1 bar Ar separately, and their mixture. The generated XUV and near-infrared (NIR) beams passed through a 100 -nm-thick Al filter, which filtered out all wavelengths corresponding to energies below $15 \mathrm{eV}\left(\omega \approx 2.3 \times 10^{16} \mathrm{rad} \mathrm{s}^{-1}\right)$. The XUV spectrum was measured by an XUV spectrometer, with a spectral threshold accepting energies above $20 \mathrm{eV}\left(\omega \approx 3 \times 10^{16} \mathrm{rad} \mathrm{s}^{-1}\right)$. Figure 2a shows the three XUV spectra associated with the two attosecond pulses and their interference. The structural difference between the ground state of $\mathrm{Ar}$ and $\mathrm{N}_{2}$ leads to the generation of two significantly different attosecond XUV pulses. The similar ionization potentials of the two gases, $I_{\mathrm{p}}^{\mathrm{Ar}}=15.8 \mathrm{eV}$ and $I_{\mathrm{p}}^{\mathrm{N}_{2}}=15.6 \mathrm{eV}$, give rise to a broad spectral overlap region over which the relative 


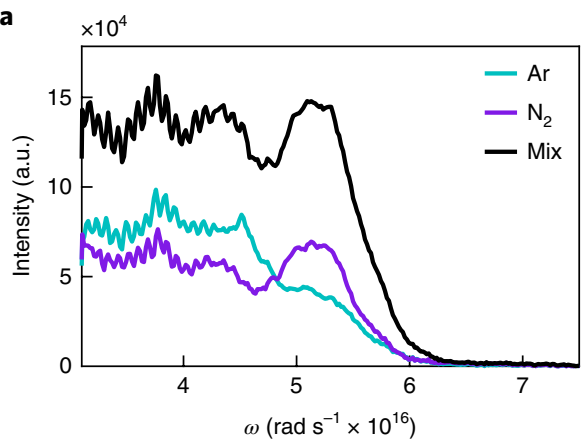

C

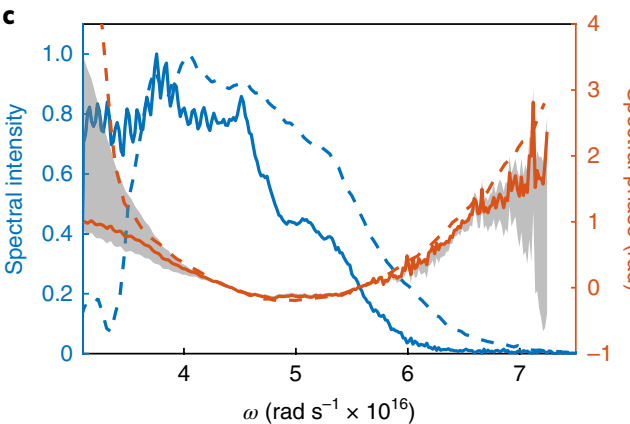

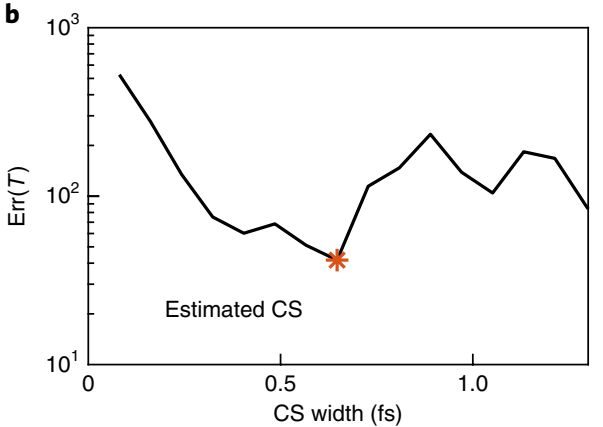

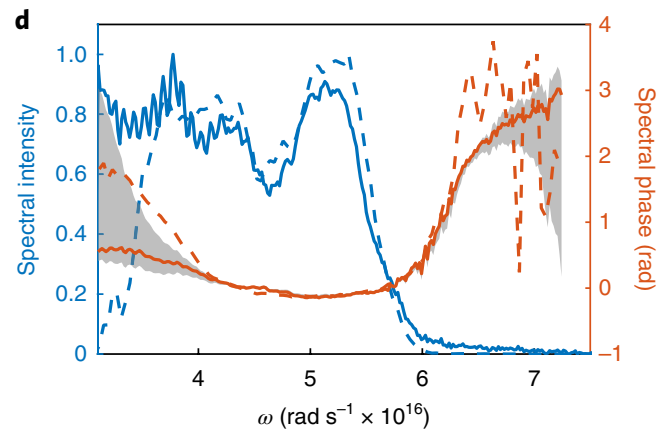

Fig. 2 | Gas mixture single-pulse DBH. a, XUV spectra from three different gases: $\mathrm{Ar}_{1} \mathrm{~N}_{2}$ and their mixture. Frequencies below $3 \times 10^{16}$ rad s ${ }^{-1}$ are not detected in our spectrometer. $\mathbf{b}$, The error score curve and the estimated compact support (CS) found at the global minimum of the curve. c,d, The normalized XUV spectral intensity (solid blue line) together with the DBH retrieved spectral phase (solid orange) at the evaluated compact support for Ar (c) and $\mathrm{N}_{2}$ (d). The grey shaded area marks the possible uncertainty in the spectral phase due to an inaccurate choice of compact support. The normalized photoelectron spectrum (dashed blue) and the FROG-CRAB retrieved phase (dashed orange) are plotted for comparison.

phase can be extracted. Using low gas density ensures that propagation effects are negligible and that the mixture spectrum indeed represents a coherent addition of the pure gas cases.

Given the three measured spectra and the extracted phase difference, we next applied the $\mathrm{DBH}$ algorithm for compact support values in the range $0-1.35 \mathrm{fs}$. We identified a global minimum in the error score curve at $T \approx 640$ as, corresponding to the estimated compact support (Fig. 2b). Figure 2c,d presents the retrieved spectral phases (solid orange lines) obtained by $\mathrm{DBH}$ at the estimated compact support, along with the original spectra for both Ar and $\mathrm{N}_{2}$ (solid blue lines). The spectral phases exhibit pronounced group delay dispersion, $\mathrm{GDD}_{\mathrm{Ar}}=8 \times 10^{-33} \mathrm{~s}^{2}$ and $\mathrm{GDD}_{\mathrm{N}_{2}}=7 \times 10^{-33} \mathrm{~s}^{2}$, and also some higher-order terms. The grey areas in Fig. $2 \mathrm{c}, \mathrm{d}$ represent the uncertainty of the reconstruction procedure. We estimate this area by choosing compact support values shifted from the minimum by \pm 80 as (one temporal resolution step). Naturally, the uncertainty grows at regions of low signal. We note that any spectral phase-only filter applied to both of the pulses will not affect the measured spectra. Such ambiguity cannot be resolved by the DBH approach ${ }^{26}$.

We validated our reconstructions by comparing the retrieved pulses with a FROG-CRAB characterization. Maintaining the same experimental conditions, we focused the unknown XUV pulses together with a NIR beam into a second neon target, where we performed the FROG-CRAB measurement. The strong NIR field here serves as a temporal gate for XUV photoionized electrons, mapping their pulse properties into their electron momenta ${ }^{8,9}$. Experimentally, we scanned the delay between the NIR and XUV fields and measured the photoelectron spectrum using an electron time-of-flight (TOF) detector (for details see Supplementary Information). Figure $2 \mathrm{c}, \mathrm{d}$ compares the photoelectron spectrum in both gases, as measured by the TOF spectrometer (dashed blue lines) and shifted by $I_{\mathrm{p}}$. The XUV and photoelectron spectra do not match perfectly, mainly due to the TOF response function. Both the neon ionization dipole and the fact that low-energy electrons are not effectively collected cause a deviation at low energies (frequencies). At low electron kinetic energies, standard FROG-CRAB algorithms fail to accurately reconstruct the attosecond pulse. To overcome this problem we combined quick and noise-robust ePIE reconstruction ${ }^{28}$ with the Volkov transform generalized projection algorithm (VTGPA) ${ }^{29}$ (see Supplementary Information for details). The FROG-CRAB spectral phase reconstruction (dashed orange lines in Fig. 2c,d), obtained after 2,000 iteration of the ePIE code and refined by 100 iterations of the VTGPA code, is in close agreement with the DBH result. Deviations appear only at very high frequencies where both signals are relatively weak and at low frequencies where the TOF spectrometer count is low. The FROG-CRAB measurement averages multiple attosecond pulse realizations, which, unfortunately, are not all identical and differ due to intensity and CEP fluctuations of the NIR field. In contrast, the linear nature of $\mathrm{DBH}$ allows for a faster characterization and enables post-selection of spectra obtained under similar experimental conditions (see Supplementary Information).

DBH of attosecond pulses is not limited to the gas mixture realization. Next, we take an important step forward, demonstrating the ability to perform DBH using a single-shot measurement. Singleshot $\mathrm{DBH}$ was first demonstrated in the spatial domain ${ }^{25}$. The diffraction of two (or more) finite objects separated by more than twice their size allows the extraction of their individual spectra and relative spectral phase directly from the autocorrelation signal. In this case the two well-separated objects serve as unknown references to one another, representing the two arms of the hologram (Fig. 1b). In this Article we demonstrate the temporal analogue of this scheme by considering two finite pulses that are delayed by more than twice their individual compact support size.

Single-shot DBH is demonstrated by tuning the CEP of the driving pulse to generate two attosecond pulses, separated by half the 

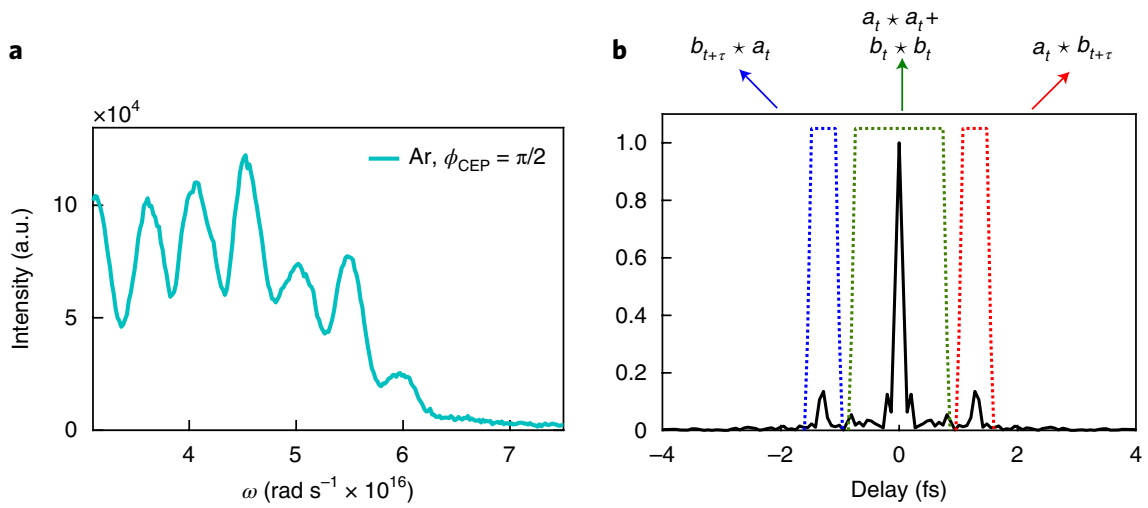

c

d
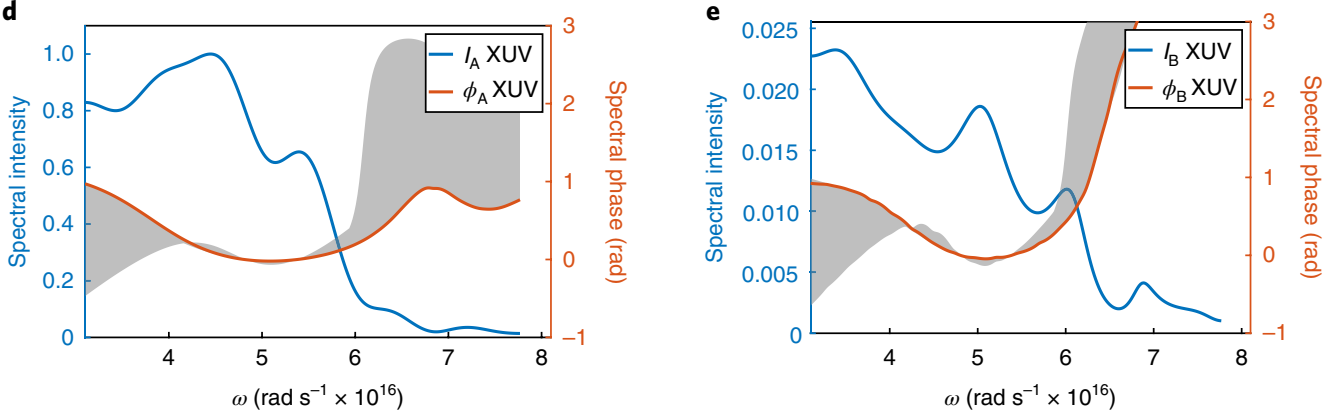

Fig. 3 | Single-shot double-pulse DBH. a, By setting the CEP phase to $\sim \pi / 2$ we generate two consecutive attosecond pulses in Ar. The two pulses interfere in the spectral domain, producing spectral fringes. $\mathbf{b}$, The autocorrelation signal (absolute value) shows the main lobe and two side peaks, separated by $1.35 \mathrm{fs}$ (half period of the driving field). The main lobe corresponds to the sum of single-pulse autocorrelations, and the side peaks correspond to crosscorrelations. We 'cut out' the different peaks according to the dashed windows and extracted the individual pulse spectra and spectral phase difference. c, The error score curve and the estimated compact support, found at the global minimum of the curve. d,e, Phase retrieval results. The normalized XUV spectral intensity (solid blue line) together with the DBH retrieved spectral phase (solid orange line) at the evaluated compact support for the two individual pulses. The grey shaded area marks the possible uncertainty in the spectral phase due to an inaccurate choice of the compact support.

optical period of the fundamental field. Figure 3a shows the intensity spectrum measured in Ar gas, showing deep spectral modulations. The modulation period corresponds to a delay between the two pulses of $1.35 \mathrm{fs}$, exceeding the expected compact support of each individual pulse, thus satisfying the basic requirement for single-shot reconstruction. Figure $3 \mathrm{~b}$ describes the autocorrelation signal, with a main peak and strong side bands arising from the single-object autocorrelations and cross-correlations, respectively. By applying a Fourier transform on the separate peaks we are able to retrieve the individual spectra of the two interfering pulses and their relative phase (see Supplementary Information).

As in the single-pulse case, we search for the optimal compact support width and identify a clear minimum at $T=490$ as (Fig. 3c). The retrieved spectral amplitudes and spectral phase, associated with each attosecond pulse, are presented in Fig. 3e,f. The difference in pulse intensities is attributed to envelope and saturation effects. The expected spectral phase chirp appears clearly in both pulses, where the grey sleeves indicate a spectral phase error arising from \pm 70 as choices of the compact support. The stronger pulse has a GDD comparable to the single-pulse case, $\mathrm{GDD}_{\mathrm{A}}=6 \times 10^{-33} \mathrm{~s}^{2}$, whereas for the weaker pulse we find a higher value, $\mathrm{GDD}_{\mathrm{B}}=1.1 \times 10^{-32} \mathrm{~s}^{2}$. This effect is dominated by the NIR pulse envelope and can be explained in the electron trajectory picture. Electron trajectories launched at the first half-cycle are driven by a strong returning force whereas trajectories launched at the second half cycle are influenced by a weaker returning force, and exhibit higher dispersion in arrival times. We approximated this effect using a classical trajectory simulation under our experimental conditions and found that the expected GDDs are $8 \times 10^{-33} \mathrm{~s}^{2}$ for the stronger pulse, and $1.3 \times 10^{-32} \mathrm{~s}^{2}$ for the weaker pulse, in good agreement with the reconstructed values. Better accuracy can be achieved by taking into account the effects of tunnelling and compression in the Al filter.

In conclusion, we have introduced a conceptually new approach to address a key challenge in ultrafast measurements-phase retrieval for ultrashort temporal signals-demonstrating a direct and linear reconstruction of attosecond pulses. DBH can be realized in a wide range of experimental schemes, from polarization measurements to transient gratings or multiple-orbital HHG. To demonstrate the versatility of $\mathrm{DBH}$, we have applied this method in two different scenarios: gas mixture and spectral interference of delayed pulses. In contrast to current time-domain nonlinear approaches, our approach is essentially a single-shot measurement. This removes the primary limitations in the characterization of a range of novel ultrafast sources, such as X-ray free-electron laser attosecond pulses or attosecond plasma mirrors. Looking forward, temporal $\mathrm{DBH}$ can be implemented to characterize such sources and allow instant attosecond pulse diagnostics. Using this new tool to study complex electron dynamics may give rise to a new class of time-resolved experiments, where attosecond-scale phenomena can be observed using a linear, single-shot measurement.

\section{Online content}

Any methods, additional references, Nature Research reporting summaries, source data, statements of data availability and associated accession codes are available at https://doi.org/10.1038/ s41566-018-0308-z.

Received: 7 September 2017; Accepted: 5 November 2018; Published online: 24 January 2019 


\section{References}

1. Paul, P. M. et al. Observation of a train of attosecond pulses from high harmonic generation. Science 292, 1689-1692 (2001).

2. Sansone, G. et al. Electron localization following attosecond molecular photoionization. Nature 465, 763-766 (2010).

3. Smirnova, O. et al. High harmonic interferometry of multi-electron dynamics in molecules. Nature 460, 972-977 (2009).

4. Diveki, Z. et al. Spectrally resolved multi-channel contributions to the harmonic emission in $\mathrm{N}_{2}$. New J. Phys. 14, 023062 (2012).

5. Calegari, F. et al. Ultrafast electron dynamics in phenylalanine initiated by attosecond pulses. Science 346, 336-339 (2014).

6. Nisoli, M., Decleva, P., Calegari, F., Palacios, A. \& Martín, F. Attosecond electron dynamics in molecules. Chem. Rev. 117, 10760-10825 (2017).

7. Garg, M. et al. Multi-petahertz electronic metrology. Nature 538, 359-363 (2016).

8. Mairesse, Y. \& Quéré, F. Frequency-resolved optical gating for complete reconstruction of attosecond bursts. Phys. Rev. A 71, 011401 (2005).

9. Sansone, G. et al. Isolated single-cycle attosecond pulses. Science 314, 443-446 (2006).

10. Mairesse, Y. et al. Attosecond synchronization of high-harmonic soft X-rays. Science 302, 1540-1543 (2003).

11. Tzallas, P., Charalambidis, D., Papadogiannis, N., Witte, K. \& Tsakiris, G. D. Direct observation of attosecond light bunching. Nature 426, 267-271 (2003).

12. Nabekawa, Y. et al. Interferometric autocorrelation of an attosecond pulse train in the single-cycle regime. Phys. Rev. Lett. 97, 153904 (2006).

13. Kim, K. T. et al. Manipulation of quantum paths for space-time characterization of attosecond pulses. Nat. Phys. 9, 159-163 (2013).

14. Mairesse, Y. et al. High harmonic XUV spectral phase interferometry for direct electric-field reconstruction. Phys. Rev. Lett. 94, 173903 (2005).

15. Levesque, J. et al. Polarization state of high-order harmonic emission from aligned molecules. Phys. Rev. Lett. 99, 243001 (2007).

16. Camper, A. et al. High-harmonic phase spectroscopy using a binary diffractive optical element. Phys. Rev. A 89, 043843 (2014).

17. Bruck, Y. M. \& Sodin, L. On the ambiguity of the image reconstruction problem. Opt. Commun. 30, 304-308 (1979).

18. Barakat, R. \& Newsam, G. Necessary conditions for a unique solution to two-dimensional phase recovery. J. Math. Phys. 25, 3190-3193 (1984).

19. Klibanov, M. V., Sacks, P. E. \& Tikhonravov, A. V. The phase retrieval problem. Inverse Probl. 11, 1 (1995).

20. Miao, J., Sayre, D. \& Chapman, H. Phase retrieval from the magnitude of the Fourier transforms of nonperiodic objects. J. Opt. Soc. Am. A 15, 1662-1669 (1998).

21. Miao, J., Ishikawa, T., Anderson, E. H. \& Hodgson, K. O. Phase retrieval of diffraction patterns from noncrystalline samples using the oversampling method. Phys. Rev. B 67, 174104 (2003).

22. Hayes, M., Lim, J. \& Oppenheim, A. Signal reconstruction from phase or magnitude. IEEE Trans. Acoustics, Speech, and Signal Processing 28, 672-680 (1980).

23. Beinert, R. \& Plonka, G. Ambiguities in one-dimensional discrete phase retrieval from Fourier magnitudes. J. Fourier Anal. Appl. 21, 1169-1198 (2015).
24. Raz, O. et al. Direct phase retrieval in double blind Fourier holography. Opt. Express 22, 24935-24950 (2014).

25. Leshem, B. et al. Direct single-shot phase retrieval from the diffraction pattern of separated objects. Nat. Commun. 7, 10820 (2016).

26. Raz, O. et al. Vectorial phase retrieval for linear characterization of attosecond pulses. Phys. Rev. Lett. 107, 133902 (2011).

27. Raz, O., Dudovich, N. \& Nadler, B. Vectorial phase retrieval of 1-D signals. IEEE Trans. Signal Processing 61, 1632-1643 (2013).

28. Lucchini, M. et al. Ptychographic reconstruction of attosecond pulses. Opt. Express 23, 29502-29513 (2015).

29. Keathley, P. D., Bhardwaj, S., Moses, J., Laurent, G. \& Krtner, F. X. Volkov transform generalized projection algorithm for attosecond pulse characterization. New J. Phys. 18, 073009 (2016).

\section{Acknowledgements}

The authors thank Y. Mairesse for discussions and helpful comments. N.D. is the incumbent of the Robin Chemers Neustein professorial Chair, and gratefully acknowledges the Minerva Foundation, the Israeli Science Foundation, the European Research Council Starting Research Grant MIDAS, the Crown Photonics Center and the I-Core Center for financial support. F.C. acknowledges financial support from ERC Starting Research Grant STARLIGHT no. 637756. D.O. acknowledges financial support from the ICore program and the Crown Photonics Center. B.N. is the incumbent of the William Petschek Professorial Chair of Mathematics, and acknowledges financial support from the Israeli Science Foundation. O.R. is the incumbent of the Shlomo and Michla Tomarin career development chair, and is supported by a research grant from $\mathrm{Mr}$ and Mrs Dan Kane and the Abramson Family Center for Young Scientists.

\section{Author contributions}

O.P., B.L., O.R., D.O., F.C. and N.D. conceived and designed the experiments. O.P., A.T., B.L., M.C.C., M.G., E.P.M., F.C., L.P. and F.F. performed the experiments. O.P., A.T., B.L, H.S., F.C., D.O. and N.D. analysed the data. O.P., A.T., H.S., M.L. and F.C. contributed materials/analysis tools. O.P., A.T., B.N., O.R., M.N., F.C., D.O. and N.D. co-wrote the paper.

\section{Competing interests}

The authors declare no competing interests.

\section{Additional information}

Supplementary information is available for this paper at https://doi.org/10.1038/ s41566-018-0308-Z.

Reprints and permissions information is available at www.nature.com/reprints. Correspondence and requests for materials should be addressed to O.P. or N.D. Publisher's note: Springer Nature remains neutral with regard to jurisdictional claims in published maps and institutional affiliations.

(c) The Author(s), under exclusive licence to Springer Nature Limited 2019 


\section{Methods}

Let $A_{n}=\left|A_{n}\right| e^{i \phi_{n}^{A}}=\sum_{t=0}^{N-1} a_{t} e^{2 \pi i t n / N}$ and $B_{n}=\left|B_{n}\right| e^{i \phi_{n}^{B}}=\sum_{t=0}^{N-1} b_{t} e^{2 \pi i t n / N}$ be the discrete Fourier transforms associated with the two ultrashort signals $\left\{a_{t}, b_{t}\right\}_{t=0}^{N-1}$, respectively. In a DBH, the spectral intensity of the two signals, $\left|A_{n}\right|^{2}$ and $\left|B_{n}\right|^{2}$, and the spectral intensity of their coherent sum, $\left|A_{n}+B_{n}\right|^{2}=\left|\sum_{t=0}^{N-1}\left(a_{t}+b_{t}\right) e^{2 \pi i t n / N}\right|^{2}$, are measured. The goal is to characterize the temporal signals $\left\{a_{t}, b_{t}\right\}_{t=0}^{N-1}$, or, equivalently, recover the missing spectral phase vectors $X_{n}^{A}=e^{i \phi_{n}^{A}}$ and $X_{n}^{B}=e^{i \phi_{n}^{B}}$, both of length $N$.

The relative spectral phases $\phi_{n}^{B}-\phi_{n}^{A}$ are extracted from the intensity measurements according to

$$
\phi_{n}^{B}-\phi_{n}^{A}=\arccos \left(\frac{\left|A_{n}+B_{n}\right|^{2}-\left|A_{n}\right|^{2}-\left|B_{n}\right|^{2}}{2\left|A_{n} B_{n}\right|}\right)
$$

In general, there exists a sign ambiguity associated with the inverse cosine branches ${ }^{30}$. However, in our specific setting such a zero crossing does not occur Equation (1) can be formulated as a set of $N$ linear equations for the unknown phase vectors $X_{n}^{A}, X_{n}^{B}$ as follows:

$$
\left|A_{n} \| B_{n}\right| X_{n}^{A}=A_{n} B_{n}^{*} X_{n}^{B}
$$

The compact support (CS) constraint of width $T$ constitutes a second set of $2 N-2 T$ linear equations for the unknown phase vectors, $X_{n}^{A}, X_{n}^{B}$, as follows:

$$
\begin{aligned}
& \frac{1}{N} \sum_{n=0}^{N-1}\left|A_{n}\right| X_{n}^{A} e^{2 \pi i t n / N}=0 \text { for } t \notin \mathrm{CS} \\
& \frac{1}{N} \sum_{n=0}^{N-1}\left|B_{n}\right| X_{n}^{B} e^{2 \pi i t n / N}=0 \quad \text { for } \quad t \notin \mathrm{CS}
\end{aligned}
$$

We solve these linear equations for each compact support value in a range of feasible compact support sizes, returning the suggested solutions $\hat{X}_{n}{ }^{A}, \hat{X}_{n}^{B}$. These solutions correspond to the following temporal signals:

$$
\begin{aligned}
& \hat{a}_{t}=\frac{1}{N} \sum_{n=0}^{N-1}\left|A_{n}\right| \frac{\hat{X}_{n}^{A}}{\left|\hat{X}_{n}^{A}\right|} e^{2 \pi i t n / N} \\
& \hat{b}_{t}=\frac{1}{N} \sum_{n=0}^{N-1}\left|B_{n}\right| \frac{\hat{X}_{n}^{B}}{\left|\hat{X}_{n}^{B}\right|} e^{2 \pi i t n / N}
\end{aligned}
$$

For each compact support guess we calculate an error score. This score expresses the normalized amount of energy leaking out of a suggested compact support:

$$
\operatorname{Err}(T)=\frac{\sum_{t>T}\left|\hat{a}_{t}\right|^{2}}{\sum_{t}\left|\hat{a}_{t}\right|^{2}}+\frac{\sum_{t>T}\left|\hat{b}_{t}\right|^{2}}{\sum_{t}\left|\hat{b}_{t}\right|^{2}}
$$

The estimated compact support and the output solution of DBH are those corresponding to the global minimum in this error score.

The above procedure is slightly modified for the two delayed pulses. The inverse Fourier transform (IFT) of the spectral interference of two pulses delayed by $\tau$ is

$$
\operatorname{IFT}\left[\left|A_{n}+e^{2 \pi i \tau n / N} B_{n}\right|^{2}\right]=a_{t} \star a_{t}+b_{t} \star b_{t}+a_{t} \star b_{t+\tau}+b_{t+\tau} \star a_{t}
$$

where $\star$ is the cross-correlation operator. When $a_{t} b_{t}$ are temporally separated, the different terms in the autocorrelation can be used to deduce $\left|A_{n}\right|^{2}+\left|B_{n}\right|^{2}$, $e^{-2 \pi i \tau n / N} A_{n} B_{n}^{*}$ and $e^{2 \pi i z n / N} A_{n}^{*} B_{n}$. The problem of recovering $\left|A_{n}\right|,\left|B_{n}\right|$ and $A_{n} B_{n}^{*}$ is equivalent to the sign ambiguity mentioned above.

\section{Data availability}

The experimental data and computer code used in this paper are available from the corresponding authors upon reasonable request.

\section{References}

30. Leshem, B., Raz, O., Jaffe, A. \& Nadler, B. The discrete sign problem: uniqueness, recovery algorithms and phase retrieval applications. Appl. Comput. Harmon. Anal. 45, 463-485 (2018). 